\title{
Design and Implementation of IoT based Smart Surveillance
}

\author{
Ms. Avanti Pawar \\ Department of electronics and telecommunication \\ Mumbai UMIT, SNDT, Women's University \\ Mumbai, India
}

\author{
Prof. Ajay Lahane \\ Department of electronics and telecommunication \\ Mumbai UMIT, SNDT, Women's University \\ Mumbai, India
}

\begin{abstract}
The Internet of Things is the collection of objects on the internet or network that humans rely on to make their lives easier. This project proposes an efficient implementation for IoT (Internet of Things) used for monitoring and controlling the home appliances via cloud. This project aims at monitoring environmental parameter of home and controlling home appliances using various sensors via internet using Wi-Fi as communication protocol and raspberry pi as server system. The main advantage of this project is small device can be part of internet so it is easy to communicate, manage and control without human interferences. Also it provides high degree of security, safety, comfort and energy saving. Proposed implementation of home automation includes Raspberry $\mathbf{P i}$ which is small, inexpensive, portable credit-size single board computer with support for a large number of peripherals and network communication like Ethernet port, USB port, HDMI port, SD card slot. This work proposes an approach to connect number of nodes to Raspberry pi through wireless channel. Each node capable to sensed environmental parameter and taking controlling action through relay i.e. ON/OFF fan, light, TV, AC accordingly as well as transmit all the data to Raspberry Pi. Cloud based platforms help to connect to the things around us so that we can access anything at any time and any place in a user friendly manner using customized portals and in built applications. Hence, cloud acts as a front end to access
\end{abstract} cloud.

Keywords - IoT; Raspberry Pi; home automation; sensors;

\section{INTRODUCTION}

The internet of things is a new era of intelligence computing and it is providing a privilege to communicate around the world. The objective of IOT is anything, anyone, anytime, anyplace, any service and any network [12]

Internet-of-Things: The internet where the existing network of internet to the computer systems will connect to the real world objects or things. Things may include any objects, home appliances, devices, vehicles, etc. And when these things connect to the internet in specific infrastructure via standard protocols then the whole system is said to be Internet of Things (loT). In other term Internet-of-Things can be defined as sensors and actuators embedded in physical objects are linked through wired and wireless networks, often using the same Internet Protocol (IP) that connects the Internet.

Internet of things coverage is very wide and includes variety of objects like smart phones, digital cameras, sensors, etc. Once all these devices are connected to each other, they enable more and more smart processes and services that support our basic needs, economies, environment, health etc. Such large number of devices connected to internet provides many kinds of services and produce huge amount of data and information [11]. There are several application domains that will be impacted by the emerging internet of things. The development of the Internet of Things will revolutionize a number of sectors, from automation, transportation, energy, healthcare, financial services to nanotechnology.

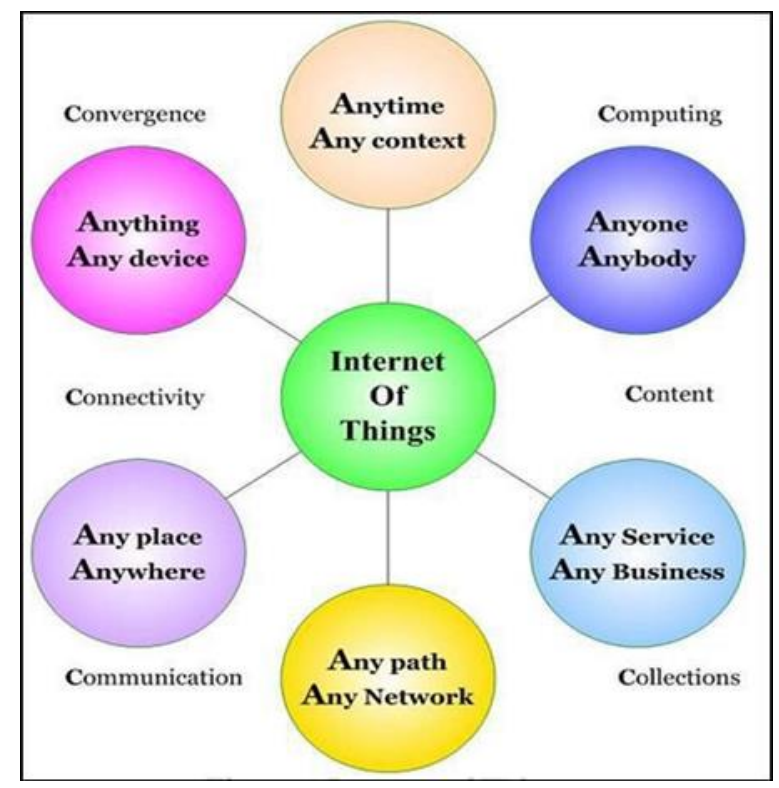

Fig. 1. Objective of IOT

Here we focus on "smart home", offering more security and energy efficiency. Home automation system required for,

- People with movement disabilities

- Older persons

- People with low vision

- Hearing impaired people

- Cognitively impaired people WHAT CAN HOME AUTOMATION DO? Home automation can:

- Increase your independence and give you greater control of your home environment.

- Save you time and effort.

- Improve your personal safety.

- Increase your home's energy efficiency.

- Alert you audibly and visually to emergency situations. 
- Allow you to monitor your home while you are away.

Here we propose to use IOT in order to control home appliances, thus automating modern homes through the internet. Not only devices can be controlled, but home environment can also be continuously monitored for maintaining certain desired temperature or monitoring amount of energy consumption [10].

\section{RELATED WORK}

[1] D. Pavithra \& Ranjith Balakrishnan have proposed IoT based monitoring and control system for home automation. This project aims at controlling home appliances via Smartphone using $\mathrm{Wi}-\mathrm{Fi}$ as communication protocol and raspberry pi as server system. The server will be interfaced with relay hardware circuits that control the appliances running at home. The communication with server allows the user to select the appropriate device. It employs IR, PIR and fire detection sensor which can detect light, presence of human being and any fire accidents respectively. This sensor sends a signal to raspberry pi. From the raspberry pi, by means of wifi configuration and IoT concept user can turn ON/OFF the light or fan or sent alert message along with the image and video taken in camera to mobile phone and an automatic phone call is made to nearby fire station as IR, PIR and fire detection sensor is triggered accordingly.

[4] Vinay sagar K N, Kusuma S M proposes a Home Automation system using Intel Galileo that employs the integration of cloud networking, wireless communication, to provide the user with remote control of various lights, fans, and appliances within their home and storing the data in the cloud. The Intel Galileo development board, with built in Wi-Fi card port to which the card is inserted, acts as web server. Automation system can be accessed from the web browser of any local PC in the same LAN using server IP, or remotely from any $\mathrm{PC}$ or mobile handheld device connected to the internet with appropriate web browser through server real IP. The model consists of different sensors like temperature, gas, motion and LDR. The designed system not only monitors the sensor data but also actuates a process according to the requirement. It also stores the sensor parameters in the cloud (Gmail) in a timely manner. This will help the user to analyze the condition of various parameters in the home anytime anywhere.

[6] Aishwarya pandey presents a brief introduction of Internet of Things (IoT) with its vision and motivation. It briefly describes its element such as RFID and WSN and puts light towards the integration of cloud and IoT. The paper even highlights the technological challenges and future directions in order to make IoT a reality. The paper also focuses on wide ranging applications of IoT

\section{PROBLEM DEFINATION}

The main objective of this project is to design a home automation system using IoT that is capable of monitoring and controlling most of the house appliances through an internet. The proposed system has a great flexibility by using wireless technology to interconnect its distributed sensors to home automation server [4]. This in turn reduces the deployment cost; will add to the flexibility of advancement, and system reconfiguration. This project aims at monitoring environmental parameter of home and controlling home appliances using various sensors via internet using $\mathrm{Wi}-\mathrm{Fi}$ as communication protocol and raspberry pi as server system. This work proposes an approach to connect number of nodes to Raspberry pi through wireless channel. Each node capable to sensed environmental parameter and taking controlling action through relay i.e. ON/OFF fan, light, TV, AC accordingly as well as transmit all the data to Raspberry Pi. Here we implement surveillance system using camera. If any suspicious moment near home is detected then camera will captures those image and update those pictures on authorized person's email automatically.

\section{HARDWARE IMPLEMENTATION}

\section{A. Proposed Methodology}

The proposed model of the home automation system is a combination of number of nodes that are transmitted sensor data to Raspberry $\mathrm{Pi}$ using wireless channel. Fig. 2 represents the block diagram of proposed model where only 1 node is shown. The main component of the system is Raspberry Pi and PIC Microcontroller. The different sensors like temperature, humidity, gas, PIR, IR connected to node1 which is PIC Microcontroller. Sensors sense the current environmental condition and send to node. Node sends those sensed value to Raspberry Pi through RF module. Raspberry Pi sent the sensor data over the internet and stored in the cloud. The data can be analyzed anywhere any time by authorized user.

In the proposed model the temperature, humidity, gas leakage and presence of human being in the house is monitored. If the sensors value goes up above the threshold value then it will controlled through relay circuit. Relay will be connected with the PIC Microcontroller which will do the work of controlling. If the temperature exceeds the threshold level then the fan will turn ON automatically and it will OFF when the temperature comes in control range. Similarly when there is a leakage of gas in the house, buzzer will raised giving the alert sound to persons inside the home. When person enter into the home PIR sensor senses this motion and light and fan will be turn ON automatically. If any suspicious moment near home is detected by IR sensor then camera will captures those image and update those pictures on authorized persons email automatically. Here Raspberry Pi will be connected to the WiFi which will send this data over the Internet by which the user will be able to see the data and can keep a watch on proper functioning of his system.

The project motto is to achieve two main applications:

(1) Monitoring

(2) Controlling 


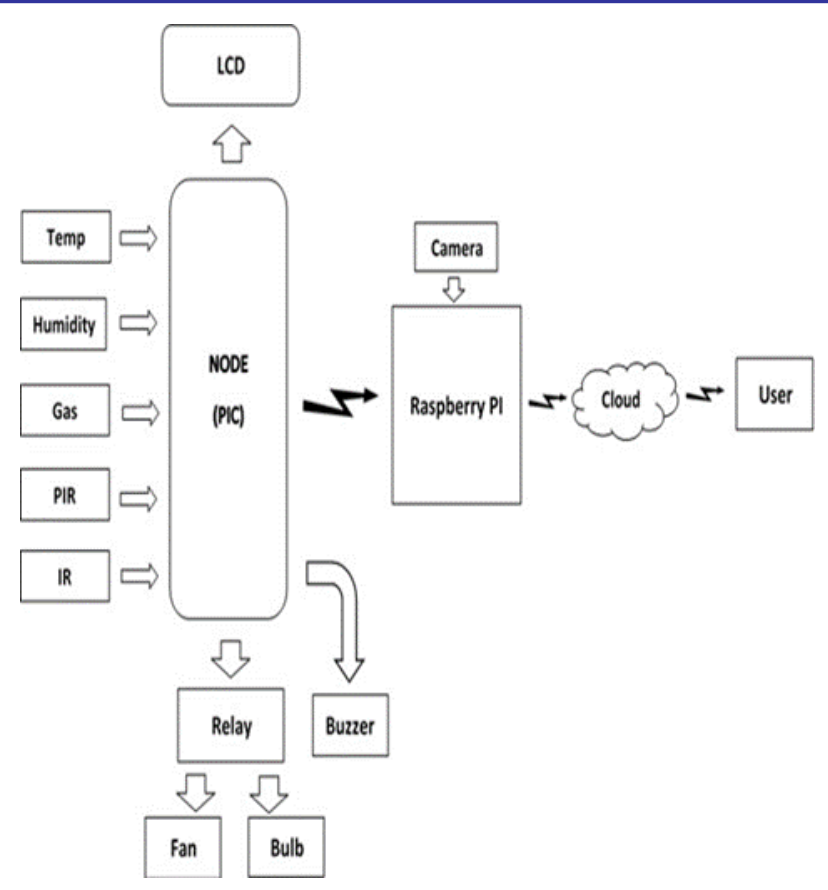

Fig. 2. Block diagram of IoT based smart surveillance

\section{B. Hardware Requirements}

The proposed home automation system has the following requirements
- Raspberry Pi Board
- PIC Microcontroller
- RF Module
- Sensor Module

\section{1) Raspberry Pi 3 Model B}

The Raspberry $\mathrm{Pi}$ is a series of small single- board computers developed in the United King-dom by the Raspberry Pi Foundation. Several generations of Raspberry Pi's have been released; Raspberry Pi 3 Model B is the newest mainline Raspberry Pi. All models feature a Broadcom system on a chip (SoC), which includes an ARM compatible central processing unit (CPU) and an on-chip graphics processing unit (GPU, a Video Core IV). CPU speed ranges from $700 \mathrm{MHz}$ to $1.2 \mathrm{GHz}$ for the Pi 3 and on board memory range from $256 \mathrm{MB}$ to $1 \mathrm{~GB}$ RAM. Secure Digital (SD) cards are used to store the operating system and program memory in either the SDHC or Micro SDHC sizes. Most boards have between one and four USB slots, HDMI and composite video output, and a $3.5 \mathrm{~mm}$ phone jack for audio. Lower level output is provided by a number of GPIO pins which support common protocols like IC. The Bmodels have an 8P8C Ethernet port and the Pi 3 and Pi Zero W have on board Wi-Fi 802.11n and Bluetooth.

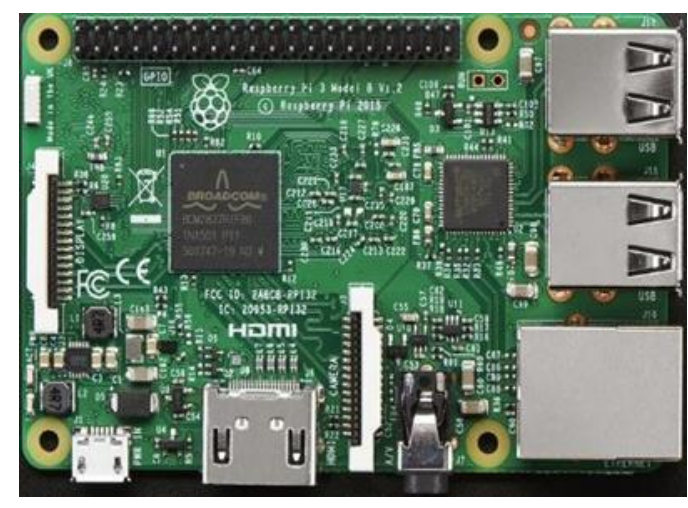

Fig. 3. Raspberry Pi 3 Model B

\section{2) PIC Microcontroller $16 F 877$}

The PIC microcontroller is based on RISC architecture. Its memory architecture follows the Harvard pattern of separate memories for program and data, with separate buses. PIC $16 \mathrm{~F} 877$ is one of the most advanced microcontroller from Microchip. This controller is widely used for experimental and modern applications because of its low price, wide range of applications, high quality and ease of availability. It is used in remote sensors, security and safety devices, home automation and in many industrial instruments

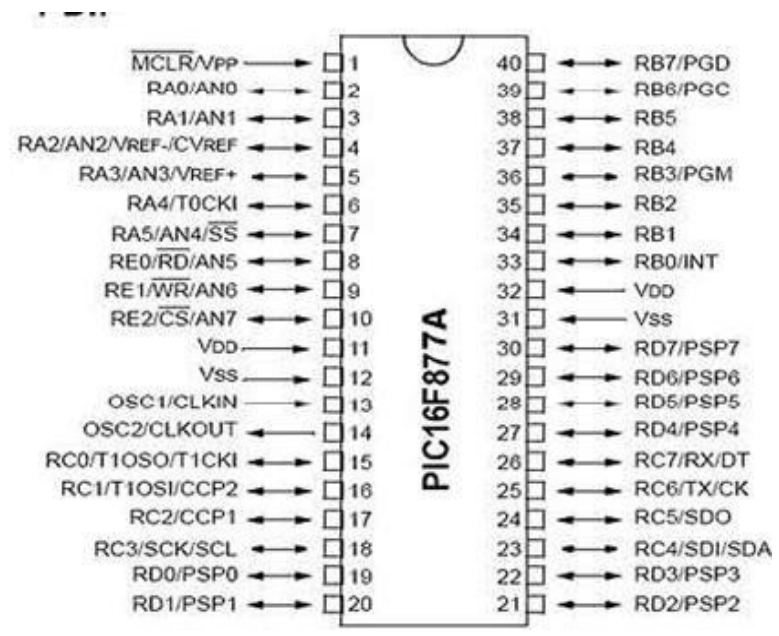

Fig. 4. Pin diagram of PIC 16F877A

\section{3) RF Module CC2500}

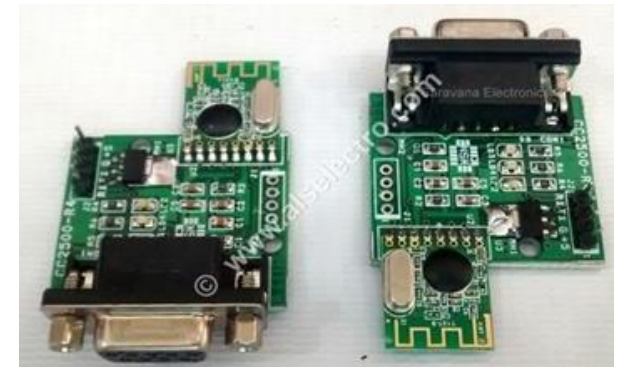

Fig. 5. RF Module CC2500

CC2500 RF Module is a transreceiver module which provides easy to use RF communication at $2.4 \mathrm{Ghz}$. It can be used to transmit and receive data at 9600 baud rates from any standard CMOS/TTL source. This module is a direct line in 
replacement for serial communication it requires no extra hardware and no extra coding to It works in Half Duplex mode i.e. it provides communication in both directions, but only one direction at same time

\section{4) Sensor Module}

\section{a) Temperature sensor LM 35}

The LM 35 series are precision integrated- circuit temperature devices with an output voltage linearly proportional to the Centigrade temperature. The LM 35 device has an advantage over linear temperature sensors calibrated in Kelvin, as the user is not required to subtract a large constant voltage from the output to obtain convenient centigrade scaling. The low-output impedance, linear output, and precise inherent calibration of the LM 35 device makes interfacing to readout or control circuitry especially easy. As the LM 35 device draws only 60 A from the supply, it has very low selfheating of less than $0.1 \mathrm{C}$ in still air. The LM 35 device is rated to operate over a $55 \mathrm{C}$ to $150 \mathrm{C}$ temperature range.

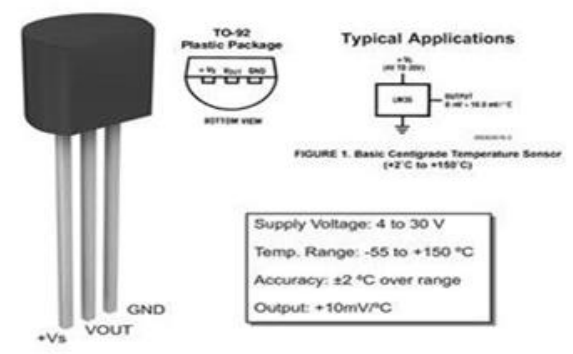

Fig. 6. Temperature sensor LM 35

\section{b) Humidity Sensor SY-HS}

A humidity sensor also called a hygrometer, measures and regularly reports the relative humidity in the air. A humidity sensor senses relative humidity. SY-HS 220 series module consists of a SYH-2 sensor and an integrated circuit to provide a linear DC voltage output for $20-95$ percentage $\mathrm{RH}$. They are specifically designed for use in appliances and controllers.

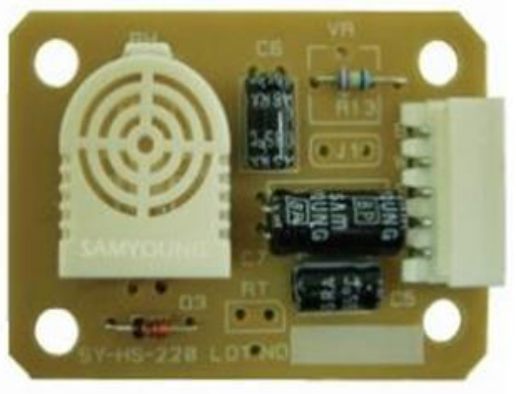

Fig. 7. SY-HS 220

c) Gas sensor MQ 2

The Analog Smoke/LPG/CO Gas sensor (MQ2) module utilizes an MQ-2 as the sensitive component and has a protection resistor and an adjustable resistor on board. The MQ-2 gas sensor is sensitive to LPG, i-butane, propane, methane, alcohol, Hydrogen and smoke. It could be used in gas leakage detecting equipments in family and industry. The resistance of the sensitive component changes as the concentration of the target gas change.

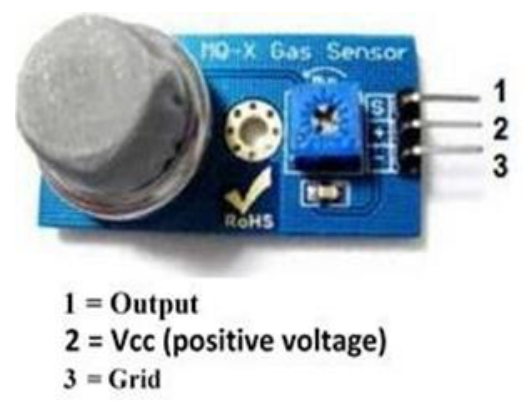

Fig. 8. Gas sensor

\section{d) PIR sensor}

A passive infrared sensor (PIR sensor) is an electronic sensor that measures infrared (IR) light radiating from objects in its field of view. The term passive in this instance refers to the fact that PIR devices do not generate or radiate any energy for detection purposes. They work entirely by detecting the energy given by other objects. PIR sensor detects a human being moving around within approximately $10 \mathrm{~m}$ from the sensor.

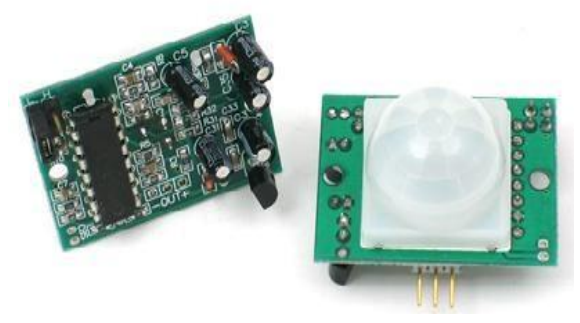

Fig. 9. PIR Sensor

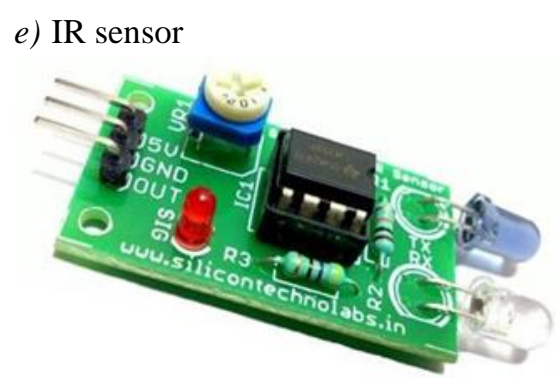

Fig. 10. IR Sensor

An infrared sensor is an electronic device that emits in order to sense some aspects of the surroundings. These types of radiations are invisible to our eyes that can be detected by an infrared sensor. The emitter is simply an IR LED (Light Emitting Diode) and the detector is simply an IR photodiode which is sensitive to IR light of the same wavelength as that emitted by the IR LED. When IR light falls on the photodiode, its resistance and correspondingly, its output voltage, change in proportion to the magnitude of the IR light received. 


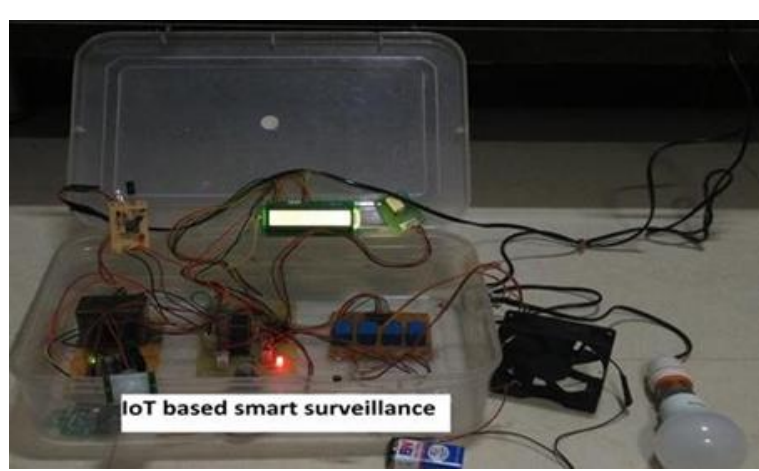

Fig. 11. Top view of IoT based smart surveillance.

\section{SOFTWARE IMPLEMENTATION}

\section{A. Monitoring}

Fig. 12 shows the flow in which the system does the monitoring of the environmental parameter of home. Sensor sense all the environment parameters and send to node i.e. PIC Microcontroller. Node sends all this information to RPI through RF Module. RPI update all this information on cloud using internet

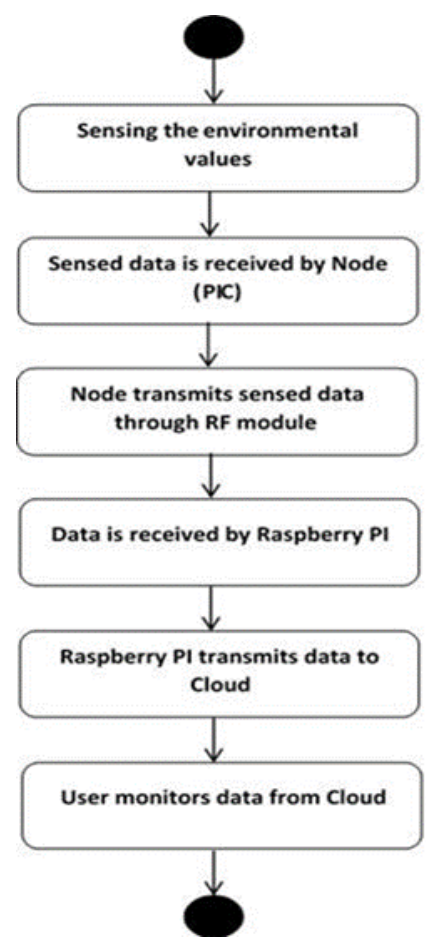

Fig. 12. Activity Diagram for monitoring the sensor value

\section{B. Controlling}

Fig. 13 demonstrates the course in which the system controls the home appliances and environment parameters. Sensed values have been sent to the PIC. PIC compares these values with the predefined threshold value as per the users need. If the measured value goes high or low the control stroke is taken by the relay for maintaining the environmental parameters in said limits. This process is repeated again and again.

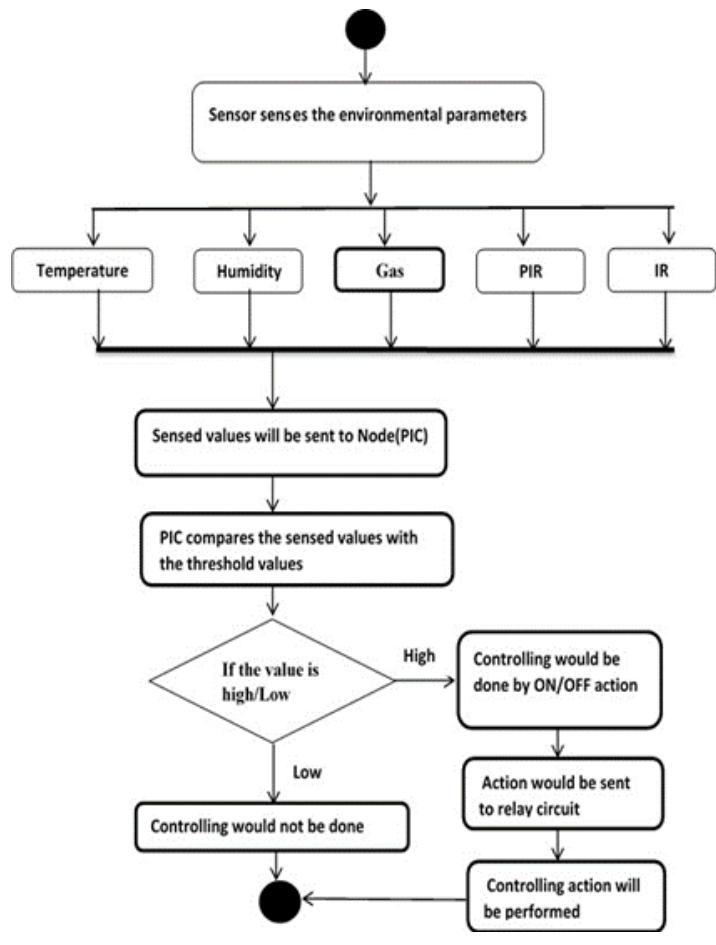

Fig. 13. Activity diagram for controlling the sensor

\section{RESULT AND ANALYSIS}

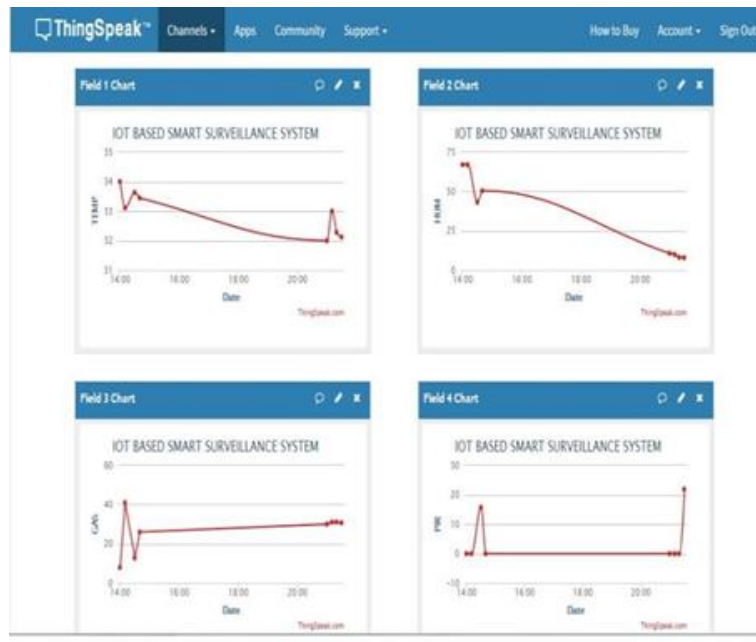

Fig. 14. Graphical representation of sensed value

The proposed home automation system is not only monitor the environmental parameters of home but also control most of the house appliances through an internet it uses RF Module to interconnect its distributed sensors to home automation server.

The RPI monitor all the sensor value and transmit all the data via Wi-Fi to cloud where it gives graphical representation of the sensor data as shown in fig. 14

The controlling action is taken by PIC itself as follows.

1) If the temperature exceeds the threshold level then the fan will turn ON

2) If humidity exceeds the threshold level then the bulb will 
glow automatically.

3) When there is a leakage of gas in the house, buzzer will raised giving the alert sound to persons inside the home.

4) When person enter into the home PIR sensor senses this motion and light and fan will be turn ON automatically.

5) If any suspicious moment near home is detected by IR sensor then camera will captures those image and RPI will update those pictures on authorized persons email automatically as shown in fig 15 .

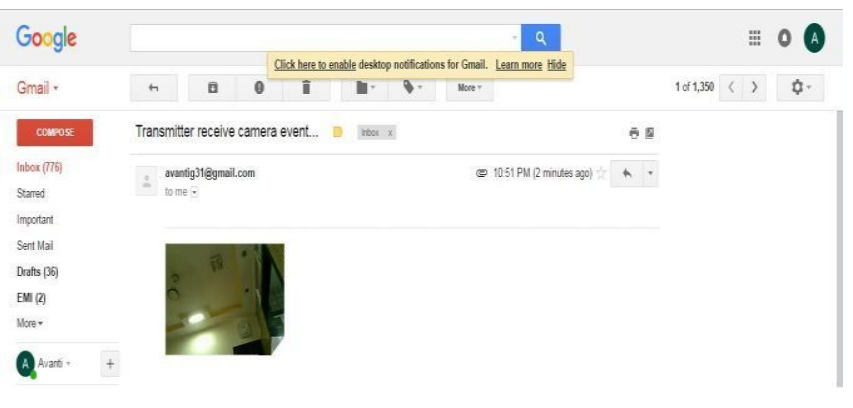

Fig. 15. E-Mail sent from Raspberry pi

\section{CONCLUSION AND FUTURE SCOPE}

In this project, we have introduced a smart home concept using Raspberry pi and wireless sensors which provides many advantages including, safety, security, improved comfort, energy and cost savings. The system allows the user to monitor various home appliances remotely anywhere in the world through an internet connection. The designed system not only monitors the sensor data, like temperature, gas, motion, but also actuates a process according to the requirement to control the parameter through relay. It also stores the sensor parameters in the cloud. This will help the user to analyze the condition of various parameters in the home anytime anywhere.
By changing the sensors this kind of system may be employed in many places like banks, labs etc. that dramatically cut back the hazard of unauthorized entry or can be implemented in the hospitals for disable people

\section{ACKNOWLEDGMENT}

It gives me a great pleasure to submit this project report on "Design and implementation of IoT based smart surveillance". This is only page where I have the opportunity to express my emotions and gratitude from the bottom of my heart. Behind every success, there is a hidden resource, the power of which is beyond compare. I am very much thankful to the most respected Prof. Ajay Lahane who contributed his valuable guidance and helped to solve every problem that arise. I am very much thankful to HOD Prof. Shikha Nema, department of E\&TC for her persistent inspiration. I would like to extend my special thanks to Principal Dr. Sanjay Pawar for spending his valuable time to go through my report and providing many helpful suggestions. Also I thank to all professors and other staff of the department for their support

\section{REFERENCES}

[1] Pavithra.D and Ranjith Balakrishnan, "IoT based monitoring and control System for home automation", In Proc. Global Conference on Communication Technologies (GCCT2015).

[2] Praveen Kumar, Umesh Chandra Pati, "IoT based monitoringand control of appliances for smart home", IEEE International Conference on Recent Trends in Electronics Information Communication Technology, May 20-21, 2016.

[3] T. Y. Ceiang and M. J. Hsiao, "Carry-select adder using single ripple carry adder,” Electron. Lett., vol. 34, no. 22, pp. 2101-2103, Oct. 1998.

[4] J. M. Rabaey, Digtal Integrated Circuits - A Design Perspective Upper Saddle River, NJ: Prentice-Hall, 2001

[5] J. M. Rabaey, Digtal Integrated Circuits - A Design Perspective Upper Saddle River, NJ: Prentice-Hall, 2001.

[6] Y. He, C. H. Chang, and J. Gu, "An area efficient 64-bit square root carry-select adder for lowpower applications," in Proc. IEEE Int. Symp. Circuits Syst., 2005, vol. 4, pp. 4082-4085.

[7] Cadence, "Encounter user guide," Version 6.2.4, March 2008 